Journal of Advanced Research in Fluid Mechanics and Thermal Sciences

Journal homepage: www.akademiabaru.com/arfmts.html ISSN: 2289-7879

\title{
Reduction of Drag Resistance by Pressure Drop in Pipeline with 3- Dimensional Design Optimization
}

\author{
Gunawan $^{1}$, Allessandro Setyo Anggito Utomo ${ }^{1,},{ }^{*}$ F. Fariz ${ }^{1}$, S. A. Lambang ${ }^{1}$ \\ 1 Department of Mechanical Engineering, Faculty of Engineering, Universitas Indonesia, Depok 16424, Indonesia
}

\section{ARTICLE INFO}

\section{Article history:}

Received 29 September 2020

Received in revised form 28 April 2021

Accepted 5 May 2021

Available online 15 June 2021

\section{Keywords:}

Optimal Pipeline Route; Drag Reduction; Pressure Drop; Dijkstra's Algorithm; 3Dimensional Projections

\section{ABSTRACT}

The searching for the most optimal pipeline route is a crucial problem in the maritime world because it consumes total designing time by $50 \%$. Also, with different types of ships increases the design complexity. The usual design process has not considered the aspect of distance, cost, obstacles, drag, and pressure reduction in the pipeline very accurately. However, along with algorithms' development to optimize pipeline design, the time can be cut by $40 \%$. This research uses computer-generated Dijkstra's algorithm to optimize pipeline design by considering several constraints in pipe spacing, the number of bends, crossings, pipeline stacks to improve drag reduction, and reducing pressure. This research was conducted to see the effect of pipe mapping on pressure drop, which is too influenced by human decisions that cannot consider bending, crossing, pipe piling, and bending of pipes that are too many to be considered by humans. Helping humans choose pipe mappings with various considerations that can affect pressure drop is advantageous because mapping helps to cut production times and produce a more efficient flow. In this study, this research aims to produce pressure drop by mapping pipes using the Djikstra algorithm by considering bending, crossing, and stacking, which are presented based on the 2 dimensional and 3-dimensional mapping. The data generated in the way of a comparison between drag reduction and pressure drop in pipe design optimization utilizing Dijkstra's and without using the Dijkstra's algorithm with 3-dimensional projections. The result shows the improvement of the drag reduction rate by $8 \%$ by decreasing pressure drop by $13 \%$.

\section{Introduction}

According to Boyd et al., [1], optimization is used to choose the best solutions to solve a problem. Regarding Dong [2], ship pipe route design aims to find the most optimal pipe routing by considering several constraints and objectives arranged in the ship's room. This process is the hardest one and takes the longest time to execute. According to Niu et al., [3], pipe routing design is considered the most crucial process in designing a ship.

\footnotetext{
* Corresponding author.

E-mail address: gunawan_kapal@eng.ui.ac.id
}

https://doi.org/10.37934/arfmts.83.2.4453 
Research on optimization algorithm has undoubtedly developed in the last few decades. Research conducted by Parashar et al., [4] ANN was used to optimize the composition present in fluid nanomaterials to predict dynamic viscosity with a $0.5 \%$ increase in performance over conventional methods. The method was done to facilitate the selection of the nanofluid with the lowest viscosity. Furthermore, another development was carried out by a similar study by Navid et al., [5] by utilizing the response surface method, which is useful for optimizing thermal conductivity using a nanomaterial in the form of a composite with the recommended fraction size from mathematical modeling. Both of these studies use mathematical methods to optimize fluid movement to be more efficient and help humans facilitate the selection of materials for this purpose. Besides, experimental physics explains the effect of optimization of pipe shape to produce more favorable fluid characteristics. Research conducted by Akeel [6] refrigerants is a figure that helps pressure drop occur in the kinematic fluid flow; this inspires this research to do something similar because pressure drop is crucial in fluid transportation in a pipe. However, it does not use a material aid but is assisted by optimizing the pipeline mapping to reduce the pressure drop generated from each different mapping using ANN. Differences also influence this pipeline's characteristics in pipe diameter, length, and direction as applied in Akeel's research [6]. It influences the study to see differences in pipe mapping that can affect pressure drop.

There are several algorithms: The Greedy Algorithm, Branch and Bound Algorithm, Genetic Algorithm, and Dijkstra Algorithm. In line with Chen [7], the Greedy Algorithm is a well-known technique for solving various problems and optimizing (minimizing or maximizing) functions with specific goals. This algorithm starts by creating all the possibilities. Branch and Bound Algorithm is a method used to solve linear integer programming problems. The decision variable will be an integer by performing the top and bottom branches stated by Raudhantul [8].

Goldberg [9] states that Genetic algorithms are algorithms that try to apply an understanding of natural evolution to problem-solving tasks (problem-solving). The algorithm approach randomly combines the best solutions in a collection to get the best solution generation. In consonance with Dijkstra [10], the Dijkstra algorithm is used to find the shortest distance between two points (start point and endpoint). In this algorithm, the shortest distance is obtained from the optimization of the graph. The graph is one part of the scientific discipline in mathematics, which consists of points and lines. This predetermined point will later be connected, which becomes the connecting line between these two points, which will become a potential solution. This algorithm continues to connect points to produce the shortest distance. The pipeline that will be generated with this algorithm is the shortest pipeline by calculating the constraint set when the calculation of this algorithm runs. Over the last few years, using the Dijkstra Algorithm, Ando and Kimura [11] could generate automatic piping route algorithms. They conclude bending and elbow as considerations for the research.

Menon [12] states that drag reduction aims to lower the pressure drop due to friction in a pipeline. This process is executed by injecting a high-molecular-weight hydrocarbon in parts per million. Drag reduction agent could perform only in turbulent flow with the low-viscosity characteristic of liquids that works best with refined petroleum products, particularly diesel, gasoline, and light crude oils. In 1956, Smith et al., [13] mention that the frictional resistance in a pipeline could be reduced effectively by injecting a drag reduction agent (DRA). Research about DRA's effect on annular gas-liquid flow was conducted in 2001 by Al-Sarkhi and Hanratty [14]. The research uses a $0.0953 \mathrm{~m}$ horizontal pipe as the medium for the flow. It showed that the concentration of polymer solution and the injection method affect the effectiveness of the DRA. In boosting the development of drag reduction technology, Norahzan et al., [15] used a transparent 
polyvinyl chloride closed-loop fluid circulation system to examine the effects of natural drag reduction of Basella Alba L. The test used was a horizontal 1.5-in pipe as the medium.

Pathfinding is the method to find the shortest route between two points by utilizing computer software. Considering the recent research, the algorithm for optimization purposes in the pathfinding technique primarily concentrates on solving a problem with two connections. In 2002, there was research for pipe-routing algorithm development by Park and Storch [16]. They formed a cell-generation method for pipe routing in a ship engine room that results in an effective and efficient generation and evaluation of the paths. This research concludes the end-forked and middle-forked form of the branch pipeline.

Meanwhile, with the pathfinding method, Kang et al., [17] optimized the pipeline route by utilizing the least cost path algorithm and Laplace smoothing considering the obstacles. Nevertheless, they did not conclude pipe diameter as a consideration for the used method. Pipe diameter could affect the cost of pipe bending, production, and crossing. Therefore, it is necessary to add pipe diameter as one of the considerations in optimizing the pipeline route. Based on the piping cost differences, the concept of sorting which pipe goes first to minimize the piping cost is also taken into consideration. Taha [18] declares that the Dijkstra Algorithm is the most streamlined method to discover the shortest way to connect two known vertices. Hence, in this research, we use the Dijkstra Algorithm for pipeline optimization and give some improvements that generate the shortest pipe route with the smallest possible bending and crossing quantities by determining it from pressure drop and friction loss contributes to the drag reduction properties.

\section{Methodology}

This study uses the Dijkstra Algorithm since it has considered the most efficient method in finding the shortest way between two known nodes. According to Ireneusz et al., [19], the Dijkstra shortest-part algorithm is a method that utilizes entirely commanded labels, which consist of positive integers or real numbers. Using partially ordered labels could be generalized if the previous labels are not decreasing - the previous label created by crossing an edge.

The diagram shown in Figure 1 illustrates the part where the optimization process uses the Dijkstra algorithm. As seen to do optimization, requires 4 data inputs, namely information about decks, pipes, penalties, and flanges. When all four data inputs are entered, an interface for the routing pipe is created. Then do the checking of the presence or absence of the oil pipeline system. After checking, the next stage of the four input data in the form of deck, pipe, penalty, and flange information is used to produce pipe routing. The Dijkstra algorithm's last step is to check whether the generated routing pipe has received the least amount of cost. If there is not yet a new pipeline and the program has the least cost, then the routing pipe will be displayed in an image.

The considered constraints in this research consist of minimizing the pipe's length, the quantity of pipe bending and crossing, considering obstacles in the engine room, and dividing the pipe holder. The reason for choosing the mentioned constraints is finding the most efficient pipe routing and continuing the previous research. This study's design process is divided into two parts: the optimization process and data input. The optimization process uses Microsoft Visual Studio software, while the data input is designed using Microsoft Excel. The algorithm used in this study is Dijkstra's algorithm added by Constraint Condition to produce the shortest pipeline, the lowest pipe cost, the least bending, and the least inter-pipe crossing. Simultaneously, the input data is the result of conversion from design in Auto-Cad to Microsoft Excel. 


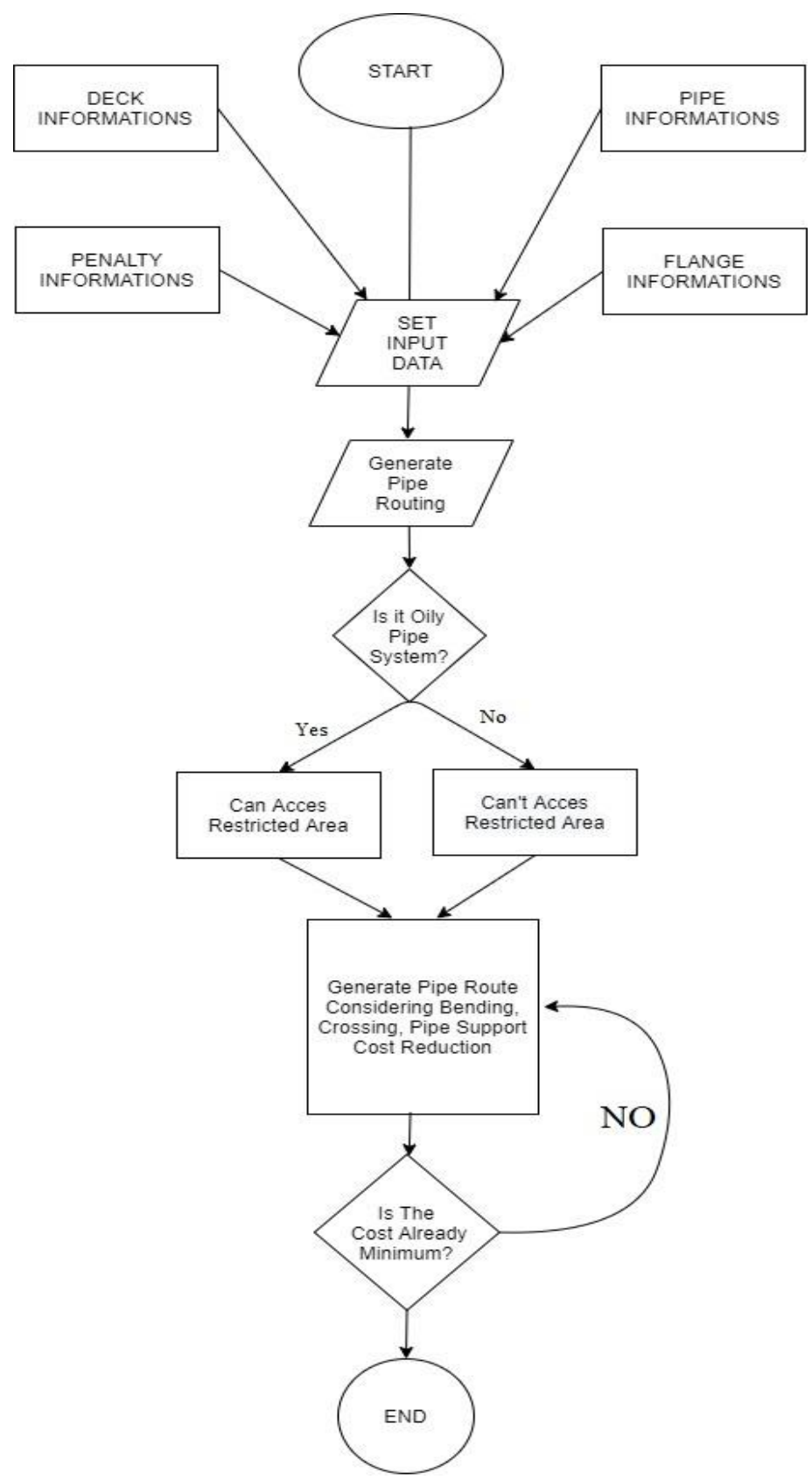

Fig. 1. Flow Diagram

Some procedures in executing the research consist of experimenting, checking, collecting optimization data, and concluding as seen in Figure 1 . The experimenting procedure starts with creating a program interface in Microsoft Visual Studio. Subsequently, the output results and limits which are wanted to be optimized were determined. After that, it determined which input data to use. Input divided into four types, namely Deck, Pipe, Flange, and Penalty. Afterward, the data regenerated from Auto-Cad to Microsoft Excel. Next, is to create a program by adding existing inputs and variables to the Dijkstra's Algorithm.

Checking conveyed regarding whether or not there are issues or bugs in the program. The issues can be seen in the toolbar at the bottom of Microsoft Visual Studio. When the bug is gone, the error message will appear, or no issues found. After that, the deck's design is being assured whether it fits its original state or not. Next, the constraints on the design are checked. 
After the program created by Microsoft Visual Studio using the Dijkstra's Algorithm has been modified, it will be run to optimize the Dijkstra's Algorithm calculation. This stage is considered as data collecting for optimization. Subsequently, after the program's optimization results are generated, the results are used to find answers to the research objectives.

According to Park [20] and Qian et al., [21], optimization of the routing pipe must solve the problem in several constraints divided into two, namely restrictive constraints and quantifiable constraints. Several constraints are divided into three, specifically physical factors, economic factors, and operational factors. Regarding physical factors, pipe routing must avoid physical barriers and be connected to the right equipment. Economic factors consist of minimizing total material and fabrication costs by reducing pipe length and the number of curves in the pipe and increasing pipe supports. Meanwhile, considering proper operation such as valve accessibility and cleaning some safety equipment is a part of operational factors. Physical factors and economic factors are considered as restrictive constraints. Hereafter, economic factors are measurable or considered as quantifiable constraints.

Furthermore, Dong [22] explains in more detail that there are many objectives and constraints in Ship Pipe Route Design (SPRD) problems. These constraints consist of connection between pipe faces

i. Avoiding obstacles and routes that if problematic (difficult)

ii. Arranging the pipes orthogonal

iii. Minimizing the pipe's length

iv. Minimizing the number of elbow bends

v. Routing the pipe near the wall or a tool for better support

vi. Dividing pipe rack if possible to reduce installation costs

vii. Arranging pipes as high in one room if possible.

Choosing the considered constraints to be inputted in the optimization procedure is necessary to generate the most efficient path for each pipeline. The chosen constraints must be relatable to the actual condition, and they will take part to prevent the occurrence of mistakes in the program utilization. This research's chosen constraints are minimizing the pipe's length, the cost of bending and crossing pipe, the cost of pipe support, and considering the obstacles in the engine room. As explained by Zong [2] on SPRD problems, there are eight main points. The optimization process is carried out on the 2D model. While in 2D models of eight SPRD points, only three points can be made, namely minimizing the length of the pipe, minimizing the number of elbow bends, and dividing the pipe rack if possible to reduce installation costs. Because this research focuses on these three points, namely minimizing the length of the pipe, minimizing the amount of bending and crossing, dividing the pipe holder, and adding calculations for the cost to get optimal results.

\section{Results}

\subsection{Input Data}

Dijkstra algorithm is used to optimize the pipeline in the engine room with a $2 \mathrm{D}$ model, and to adjust the place where the research was carried out, the researcher owned the ship's data. The ship engine room is made on a grid measuring $80 \times 48$. Assuming each grid on the ship represents 500 milli-meters on the actual ship. The input used for the grid is taken from Microsoft Excel, as shown in Figure 2. 


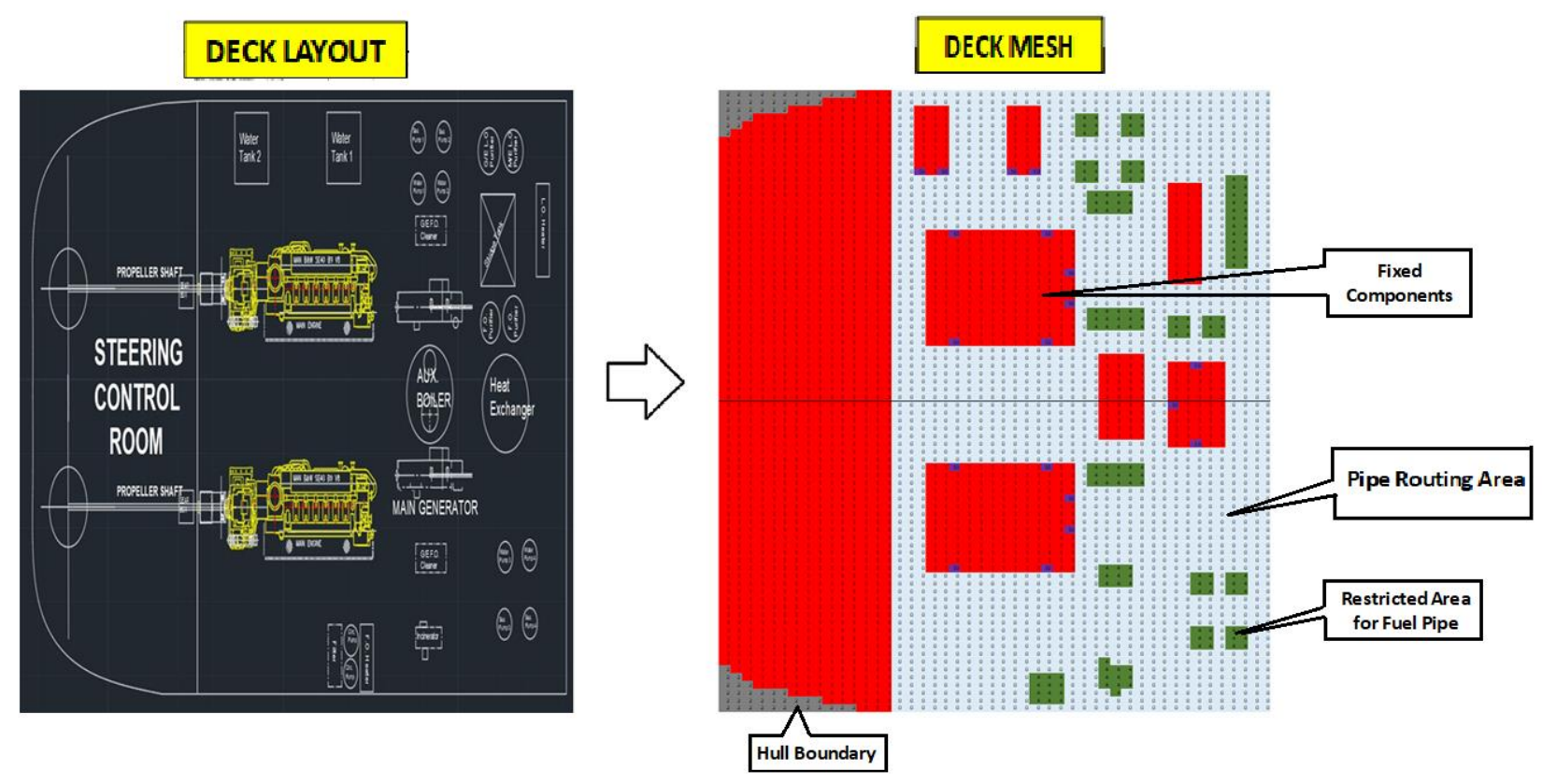

Fig. 2. Deck conversion

\subsection{Result}

Dijkstra algorithm is used to do optimization on 2-dimensional (2D) models as shown in Figure 3 and 4. After obtaining optimization on 2D models, a conversion is made to the 3D model in Figure 5.

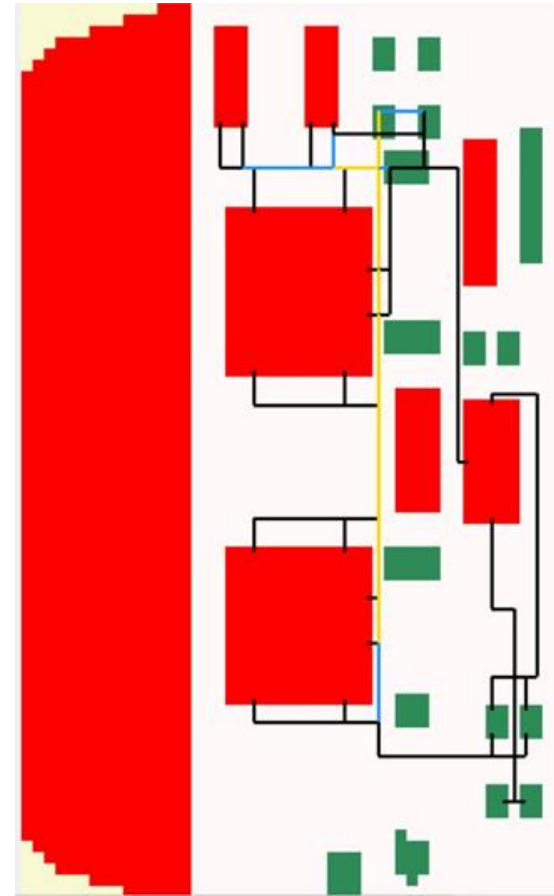

Fig. 3. Actual Pipe routing

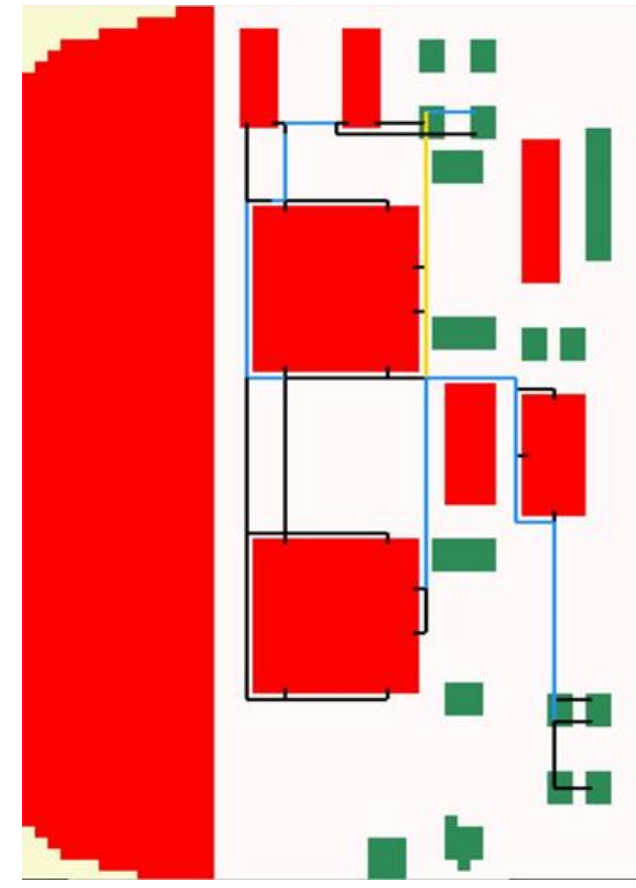

Fig. 4. After Optimization 


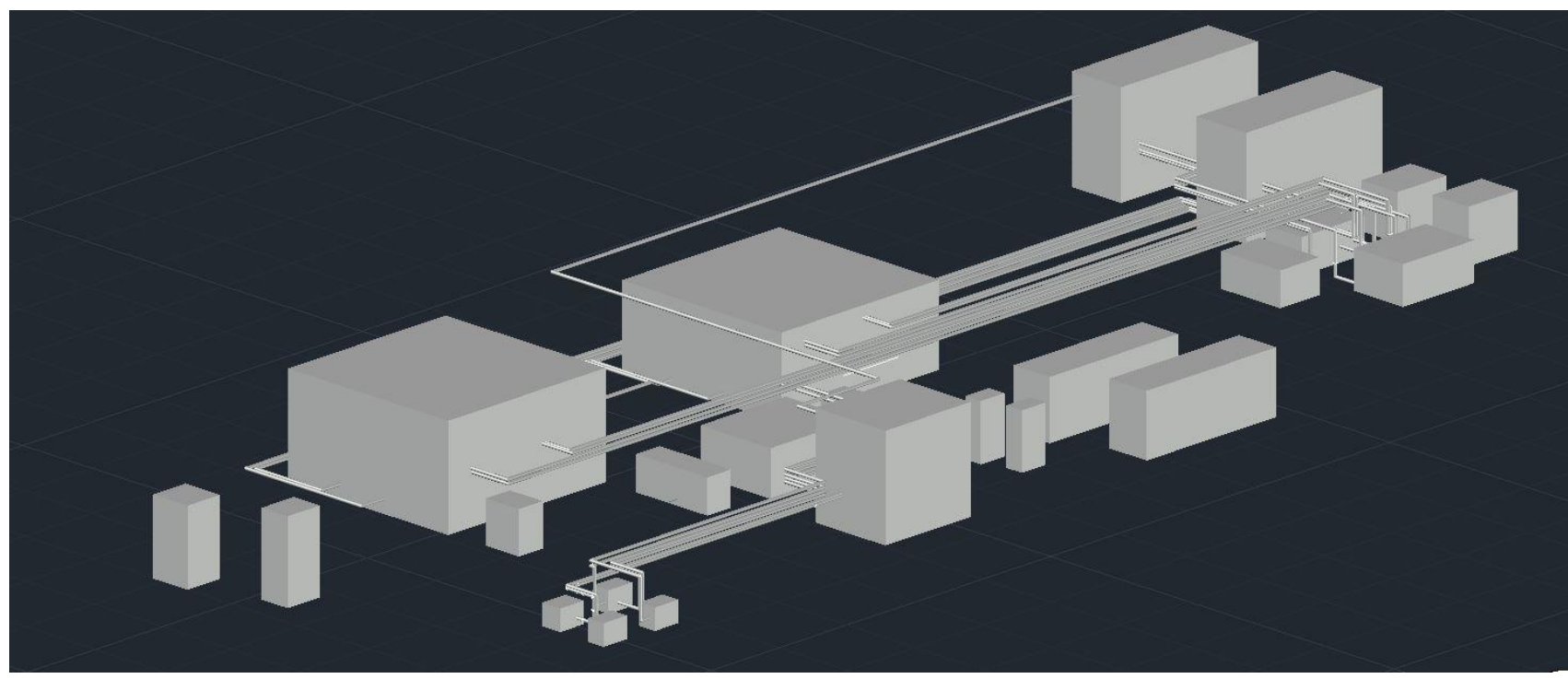

Fig. 5. 3D model pipe routing after optimization

Pipelines in 2D models have different colors. The color difference represents the total pipe diameter. The black pipe means the total diameter of the pipe is between $0-300 \mathrm{~mm}$. On the other hand, the blue color means the pipe's total diameter is between 301-600 mm. The gold color means the pipe's total diameter is between $601-1200 \mathrm{~mm}$, while a red pipeline will represent the total diameter of more than $1200 \mathrm{~mm}$.

Meanwhile, 2-dimensional models only provide information about the total number of diameter pipes in one pipeline. For getting a specific number of pipes in one path, 3-dimensional projection models are used. In addition to getting the optimization path, the Dijkstra algorithm is also used to calculate pipe length, total cost, number of bends, and crossings.

The results of the data in Table 1 will be used to calculate drag reduction and pressure drop. As for the calculation of drag reduction and pressure drop using the following formula according to the calculation of Fang et al., [23]

Table 1

Comparison of results between actual and optimization

\begin{tabular}{lll}
\hline Category & Actual & Optimization \\
\hline Pipe Length & 708 & 707 \\
Total Cost & 128583 & 118726 \\
Bending & 51 & 49 \\
Crossing & 13 & 8 \\
\hline
\end{tabular}

Formula friction factor

$\frac{1}{\sqrt{f}}=-2 \log _{10}\left(\frac{e}{3,7 D}+\frac{2,51}{R \sqrt{f}}\right)$

where $f=$ drag reduction, $e=$ absolute pipe roughness, $\mathrm{D}=$ diameter pipe, $\mathrm{R}=$ Reynold number of flow. With $D=30 \mathrm{~mm}, \mathrm{e}=0.15 \mathrm{~mm}$ (Galvanized iron), $\mathrm{R}=10000$. After the calculation, the $f$ value of 0,037231 is obtained. Beij [24] said that the length of the pipe and the amount of bending affect the friction factor because it is assumed to decrease $10 \%$ in the friction factor. This meant that by decreasing the friction factor, the simulation suggest a reduction of drag that occurred when the 
pressure is dropping. The relation between pressure drop and friction factor is written below the formula pressure drop

$J=\frac{f L v^{2}}{2 g D}$

where $\mathrm{J}=$ pressure drop, $f=$ friction factor, $\mathrm{L}=$ length of the pipe, $\mathrm{v}=$ velocity of the fluid, $\mathrm{g}=$ acceleration due to gravity. With $f=0,037231, \mathrm{v}=20 \mathrm{~m} / \mathrm{s}, \mathrm{g}=9.8 \mathrm{~m} / \mathrm{s}^{2}, \mathrm{~L}=$ based on pipe length. The calculation is carried out under two conditions: actual and optimization. After the calculation is obtained from the following data, it is then shown in Figure 6 and 7.

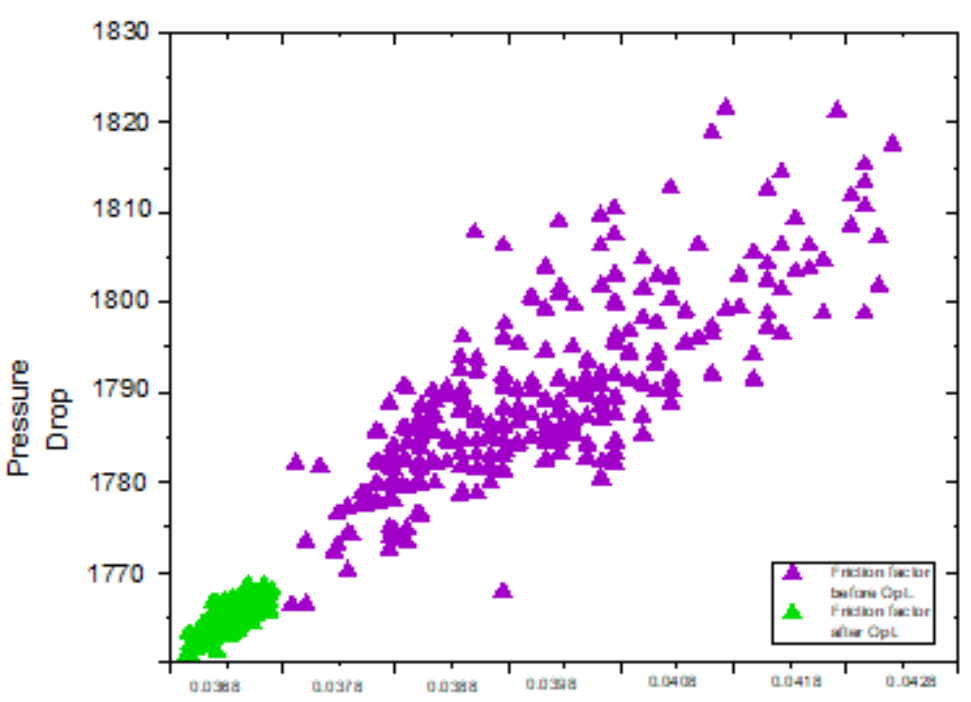

Friction Factor

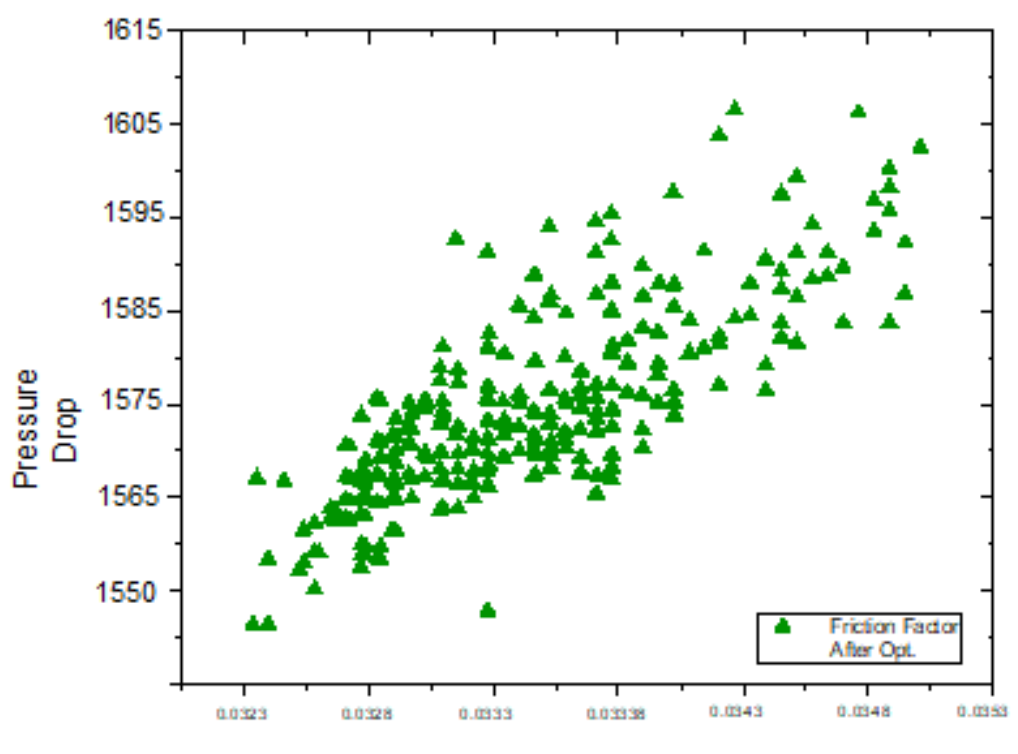

Friction Factor

Fig. 6. Pressure drop and friction factor before and after optimization 


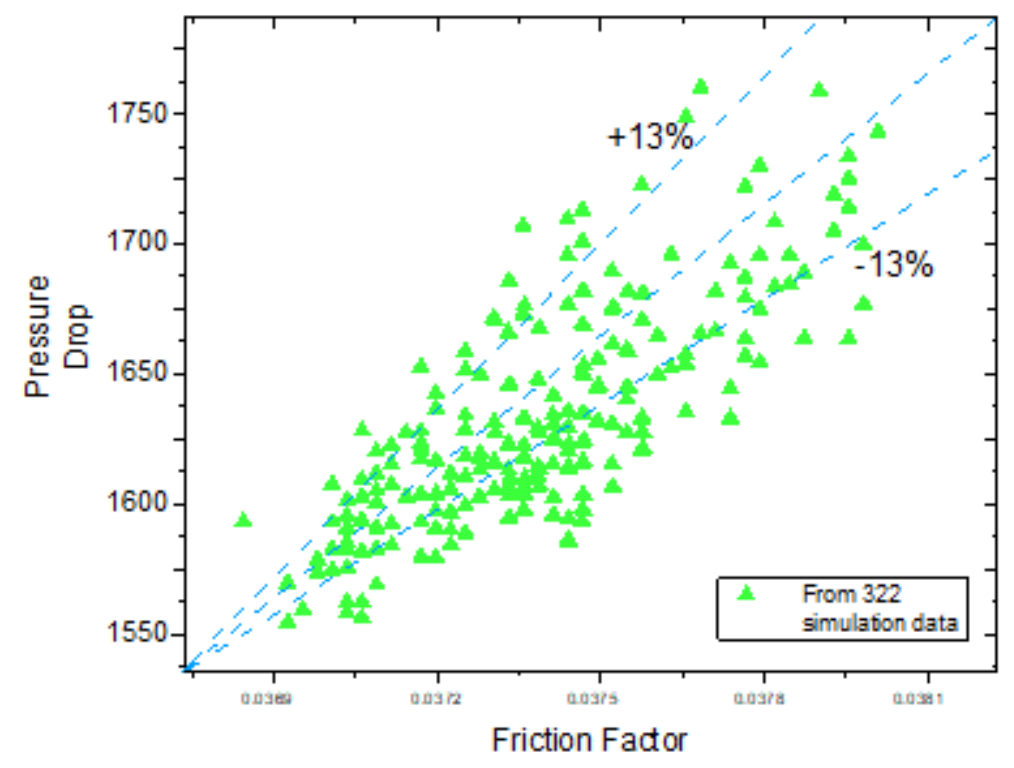

Fig. 7. Pressure drop ratio before and after optimization

Figure 6 this shows that in the calculation of data before optimization, the lowest pressure drop is found at the friction factor value of 0.00369 . The most considerable pressure drop value occurs when the friction factor value is 0.03798 . Figure 6 it also shows that the smallest pressure drop occurs when the friction factor is 0.0367 , and the largest pressure drop occurs when the friction factor is 0.0365 when after optimization. Figure 7 shows the comparison process between pre-drop before and after optimization. Before optimization, the lowest pressure drop is 1775pa. After optimization, the lowest pressure drop is 1558pa, for the highest pressure drop value before the optimization is at 1811pa, while after optimization, the pressure drop value is at 1593pa. The average pressure drop difference between before and after optimization, it was found that there was a decrease of $13 \%$. The decrease in pressure drop is also an understatement Biej [24].

\section{Conclusions}

This research investigates the conditions before and after optimization. Based on the results and data obtained, several conclusions can be drawn. Optimization occurs on the pipe's cost, the length of the pipe, the amount of bending, and the crossing amount. Then in the state before the optimization, the lowest pressure drop occurs when the friction number is 0.00368 and the highest when the friction number is 0.00377 . While after optimization, the lowest pressure drop is when the friction number is 0.00324 , and the highest is when the friction number is 0.00332 . Because the difference in the friction number affects the pressure drop, after calculating the pressure drop, is a $13 \%$ reduction. They are therefore reducing energy to transport any liquid through di pipe with minimal friction in the pipe.

\section{Acknowledgement}

This research is sponsored by Universitas Indonesia PUTI Q3 program 2020 (NKB2022/UN2.RST/HKP.05.00/2020) 


\section{References}

[1] Boyd, Stephen, Stephen P. Boyd, and Lieven Vandenberghe. Convex optimization. Cambridge university press, 2004. https://doi.org/10.1017/CBO9780511804441

[2] Dong, Zong-ran, and Yan Lin. "A particle swarm optimization based approach for ship pipe route design." International Shipbuilding Progress 63, no. 1-2 (2017): 59-84.

[3] Niu, Wentie, Haiteng Sui, Yaxiao Niu, Kunhai Cai, and Weiguo Gao. "Ship pipe routing design using NSGA-II and coevolutionary algorithm." Mathematical Problems in Engineering $2016 \quad$ (2016). https://doi.org/10.1155/2016/7912863

[4] Parashar, Naman, Navid Aslfattahi, Syed Mohd Yahya, and R. Saidur. "An artificial neural network approach for the prediction of dynamic viscosity of MXene-palm oil nanofluid using experimental data." Journal of Thermal Analysis and Calorimetry (2020): 1-12. https://doi.org/10.1007/s10973-020-09638-3

[5] Zendehboudi, Alireza, Navid Aslfattahi, Saidur Rahman, Mohd Faizul Mohd Sabri, Suhana Mohd Said, A. Arifutzzaman, and Nor Azwadi Che Sidik. "Optimization of Thermal Conductivity of NanoPCM-Based Graphene by Response Surface Methodology." Journal of Advanced Research in Fluid Mechanics and Thermal Sciences 75, no. 3 (2020): 108-125. https://doi.org/10.37934/arfmts.75.3.108125

[6] Morad, Akeel Mohammed Ali. "A Two? Phase Pressure Drop Model for Homogenous Separated Flow for Circular Tube Condenser, Examined with Four Modern Refrigerants." Journal of Advanced Research in Fluid Mechanics and Thermal Sciences 52, no. 2 (2018): 274-287.

[7] Chen, Mao. "A greedy algorithm with forward-looking strategy." In Greedy Algorithms. IntechOpen, 2008.

[8] AM, Raudhatul Jannah, Arnellis Arnellis, and Riry Sriningsih. "Optimasi Hasil Produksi Tahu dan Tempe dengan Metode Branch and Bound dan Metode Cutting Plane." UNP Journal of Mathematics 1, no. 1 (2018).

[9] Goldberg, David E., and John Henry Holland. "Genetic algorithms and machine learning." (1988).

[10] Dijkstra, Edsger W. "A note on two problems in connexion with graphs." Numerische mathematik 1, no. 1 (1959): 269-271. https://doi.org/10.1007/BF01386390

[11] Ando, Yuto, and Hajime Kimura. "An automatic piping algorithm including elbows and bends." Journal of the Japan Society of Naval Architects and Ocean Engineers 15 (2012): 219-226. https://doi.org/10.2534/ijasnaoe.15.219

[12] Menon, E. Shashi, and Pramila S. Menon. Liquid Pipepline Hudraulics. Trafford Publishing, 2013. https://doi.org/10.1201/9780203021385

[13] Smith, Robert Vincent, J. S. Miller, and J. W. Ferguson. Flow of natural gas through experimental pipe lines and transmission lines. No. BM-Mon-9. Bureau of Mines, Bartlesville, OK (USA), 1956.

[14] Al-Sarkhi, A., and T. J. Hanratty. "Effect of drag-reducing polymers on annular gas-liquid flow in a horizontal pipe." International journal of multiphase flow 27, no. 7 (2001): 1151-1162.

[15] Abdulbari, Hayder A., and Rosli Mohd Yunus. "A new natural drag reducing agent." In 2012 IEEE Colloquium on Humanities, Science and Engineering (CHUSER), pp. 631-636. IEEE, 2012.

[16] Park, Jin-Hyung, and Richard L. Storch. "Pipe-routing algorithm development: case study of a ship engine room design." Expert Systems with Applications 23, no. 3 (2002): 299-309. https://doi.org/10.1016/S09574174(02)00049-0

[17] Kang, Ju Young, and Byung Suk Lee. "Optimisation of pipeline route in the presence of obstacles based on a least cost path algorithm and laplacian smoothing." International Journal of Naval Architecture and Ocean Engineering 9, no. 5 (2017): 492-498. https://doi.org/10.1016/j.ijnaoe.2017.02.001

[18] Taha, Hamdy A. "Operations research: an introduction (Vol. 790)." (2011).

[19] Szcześniak, Ireneusz, Andrzej Jajszczyk, and Bożena Woźna-Szcześniak. "Generic Dijkstra for optical networks." Journal of Optical Communications and Networking 11, no. 11 (2019): 568-577. https://doi.org/10.1364/JOCN.11.000568

[20] Park, Jin-Hyung, and Richard L. Storch. "Pipe-routing algorithm development: case study of a ship engine room design." Expert Systems with Applications 23, no. 3 (2002): 299-309. https://doi.org/10.1016/S09574174(02)00049-0

[21] Qian, Xiao-long, Tao Ren, and Cheng-en Wang. "A survey of pipe routing design." In 2008 Chinese Control and Decision Conference, pp. 3994-3998. IEEE, 2008. https://doi.org/10.1109/CCDC.2008.4598081

[22] Dong, Zong-ran, and Yan Lin. "A particle swarm optimization based approach for ship pipe route design." International Shipbuilding Progress 63, no. 1-2 (2017): 59-84.

[23] Fang, Xiande, Yu Xu, Xianghui Su, and Rongrong Shi. "Pressure drop and friction factor correlations of supercritical flow." Nuclear Engineering and Design 242 (2012): 323-330. https://doi.org/10.1016/j.nucengdes.2011.10.041

[24] Keulegan, Garbis Hovannes, and Karl Hilding Beij. Pressure losses for fluid flow in curved pipes. US Government Printing Office, 1937. https://doi.org/10.6028/jres.021.001. 